\title{
Interação medicamentosa em pacientes com câncer de mama: uma revisão
}

\section{integrativa}

\author{
Drug interaction in breast cancer patients: an integrative review \\ Interacción farmacológica en pacientes con cáncer de mama: una revisión integradora
}

Recebido: 30/09/2021 | Revisado: 09/10/2021 | Aceito: 28/11/2021 | Publicado: 05/12/2021

\author{
Simone Cristina Pinheiro da Costa \\ ORCID: https://orcid.org/0000-0001-9499-5712 \\ Universidade Federal do Pará, Brasil \\ E-mail: simonecrissena@yahoo.com.br \\ Ana Cristina Lo Prete \\ ORCID: https://orcid.org/0000-0002-2874-5296 \\ Universidade São Judas Tadeu, Brasil \\ E-mail analoprete@gmail.com \\ Carolina Heitmann Mares Azevedo Ribeiro \\ ORCID: https://orcid.org/0000-0002-9457-2733 \\ Universidade Federal do Pará, Brasil \\ E-mail: chribeiro@ufpa.br
}

\begin{abstract}
Resumo
O câncer de mama é uma doença causada pela multiplicação desordenada de células da mama. Esse processo gera células anormais que se multiplicam, formando um tumor. Foi realizada uma revisão integrativa da literatura com o objetivo de caracterizar as pesquisas produzidas com o tema interação medicamentosa em pacientes com câncer de mama. O levantamento bibliográfico abrangeu estudos realizados no Brasil, não havendo restrições quanto ao ano de publicação dos trabalhos, em periódicos científicos e que estivessem adequados ao tema proposto para o trabalho, no período de agosto a setembro de 2021 nas bases de dados PubMed, SCIELO e BVS, utilizando os seguintes descritores: "Interações de Medicamentos", "câncer de mama" e "Brasil". Sendo identificados, após análise, 9 artigos que compuseram a amostra do estudo. Os resultados apontam que na maioria dos artigos analisados, a interação medicamentosa em pacientes com câncer, apresenta-se como principal problema relacionado a medicamentos. Em todos perceber-se a importância de se monitorar as prescrições e trabalhar em conjunto com a equipe multiprofissional, sendo o profissional farmacêutico fundamental para o cuidado as pacientes com câncer de mama.
\end{abstract}

Palavras-chave: Interações de medicamentos; Câncer de mama; Brasil.

\begin{abstract}
Breast cancer is a disease caused by the disordered multiplication of breast cells. This process generates abnormal cells that multiply, forming a tumor. An integrative literature review was carried out with the aim of characterizing the research produced with the theme drug interaction in patients with breast cancer. The bibliographic survey covered studies carried out in Brazil, with no restrictions on the year of publication of the works, in scientific journals and that were adequate to the theme proposed for the work, in the period from August to September 2021 in the PubMed, SCIELO and VHL, using the following descriptors: "Drug Interactions", "breast cancer" and "Brazil". After analysis, 9 articles were identified that made up the study sample. The results show that in most of the articles analyzed, drug interaction in cancer patients is presented as the main problem related to drugs. In all, the importance of monitoring prescriptions and working together with the multidisciplinary team is perceived, with the pharmacist being fundamental for the care of patients with breast cancer.
\end{abstract}

Keywords: Drug interactions; Breast cancer; Brazil.

\section{Resumen}

El cáncer de mama es una enfermedad causada por la multiplicación desordenada de las células de la mama. Este proceso genera células anormales que se multiplican y forman un tumor. Se realizó una revisión integradora de la literatura con el objetivo de caracterizar la investigación producida con el tema Interacción farmacológica en pacientes con cáncer de mama. El relevamiento bibliográfico abarcó estudios realizados en Brasil, sin restricciones en el año de publicación de los trabajos, en revistas científicas y que se adecuaran a la temática propuesta para el trabajo, en el período de agosto a septiembre de 2021 en PubMed, SCIELO. y BVS, utilizando los siguientes descriptores: "Interacciones medicamentosas", "cáncer de mama" y "Brasil". Tras el análisis, se identificaron 9 artículos que componían la muestra de estudio. Los resultados muestran que en la mayoría de los artículos analizados, la interacción farmacológica en pacientes con cáncer se presenta como el principal problema relacionado con las drogas. Con todo, 
se percibe la importancia del seguimiento de las prescripciones y del trabajo conjunto con el equipo multidisciplinar, siendo el farmacéutico fundamental para la atención de las pacientes con cáncer de mama.

Palabras clave: Interaciones medicamentosas; Câncer de mama; Brasil.

\section{Introdução}

O câncer de mama é uma doença causada pela multiplicação desordenada de células da mama. Esse processo gera células anormais que se multiplicam, formando um tumor. Existem vários tipos de câncer de mama, cada um possui características próprias. Alguns têm o desenvolvimento rápido enquanto outros possuem um crescimento mais lento. Dentre os diversos tipos de câncer de mama, cerca de 1\% acomete homens (INCA, 2020).

O câncer de mama é, provavelmente, a neoplasia mais temida pelas mulheres, devido às consequências do tratamento e aos efeitos psicológicos que afetam a sexualidade e a imagem pessoal (Machdo, 2017). O tratamento do câncer pode ser realizado por meio de diferentes terapias: cirurgia e radioterapia, sendo considerados tratamentos locais; quimioterapia e a terapia com agentes biológicos. A quimioterapia usada isoladamente ou em combinação tem como objetivo tratar os tumores malignos, sendo os efeitos adversos frequentes, pois são utilizadas combinações de vários sistemas quimioterápicos adjuvantes, aumentando o risco de interações (Farias et al., 2018).

Interação medicamentosa é uma resposta farmacológica ou clínica à administração de uma combinação de medicamentos, diferente dos efeitos de dois ou mais agentes dados individualmente (Do Amaral et al., 2018). O resultado final pode aumentar ou diminuir os efeitos dos princípios ativos, assim como pode promover o aparecimento de um novo efeito que não ocorreu com um dos princípios ativos sozinhos (Faria et al., 2018). As não conformidades relativas às associações de medicamentos podem ser do tipo Interações Farmacocinéticas (alteração da velocidade ou a extensão de absorção, distribuição, biotransformação ou excreção de outro fármaco), ou Interações Farmacodinâmicas (o efeito resulta da ação dos fármacos envolvidos no mesmo receptor ou enzima - sinergismo ou antagonismo) (Quemel et al., 2021)

Entre as interações medicamentosas envolvendo antineoplásicos, ciclofosfamida e fluoruracila foram os mais prevalentes. A interação entre ciclofosfamida e antieméticos antagonistas do receptor da serotonina (como a ondansetrona) foi descrita em um trabalho revisional que orienta a escolha do antiemético, baseada na eficácia, segurança e no risco de interações medicamentosas (Faria et al., 2018).

O uso de vários medicamentos simultaneamente é um dos principais fatores de risco para ocorrência de interações medicamentosas e reações adversas ao medicamento (Lima et al., 2021). As estimativas de ocorrência de interação medicamentosa a medicamentos estão entre 3\% a 5\%, em pacientes que fazem uso de dois a nove fármacos, e de $20 \%$ entre aqueles que fazem uso de 10 a 20 medicamentos, simultaneamente. Dados mais antigos mostram resultados distintos, mas que corroboram com a premissa de que o risco de interação aumenta com o número de medicamentos utilizados (Yamagata et al., 2018; Topaloğlu \& Özaslan, 2020).

Os erros mais comuns ocorridos durante o tratamento quimioterápico envolvem dose, tipo ou volume do diluente, omissão de medicamentos, instrução de hidratação e um decimal no local errado, cerca de 39\% ocorrem na prescrição, $12 \%$ na transcrição e $36 \%$ na administração (Lima et al., 2021).

As interações medicamentosas provocam efeitos prejudiciais à saúde do paciente, como intoxicação medicamentosa, efeito nulo do medicamento, não tratamento da doençae ênfase nos efeitos adversos daqueles medicamentos. O risco de reações adversas quando são administrados dois medicamentos é de 13\%, cinco medicamento é de 58\% e sete medicamentos ou mais é de $82 \%$. A falta de atenção a esse0 evento pode desencadear um impacto no tratamento, que podem ser leve, moderado grave e por fim pode levar o indivíduo à hospitalização (Lobo et al., 2021).

Portanto, o risco de interação medicamentosa cresce proporcionalmente ao número de fármacos prescritos ao paciente, e, caso este se encontre hospitalizado, os riscos aumentam devido a polifarmácia. Neste sentido, em unidades clínicas 
hospitalares, este tema merece enfoque especial, visto que a polifarmácia, somada à gravidade e instabilidade do quadro clínico dos pacientes, constitui-se em um fator extremamente relevante para a sua vulnerabilidade. (Yamagata et al., 2018)

Nesse contexto, medidas são necessárias para amenizar os riscos advindos das interações medicamentosas, ainda que, em alguns casos, tais interações sejam desejáveis. Autores sugerem revisão da farmacoterapia (incluindo fármacos antineoplásicos, terapias complementares e alternativas, bem como automedicação) com intuito de reduzir o número de medicamentos; identificação prévia de interações potenciais e manejo clínico prospectivo adequado; ações interdisciplinares envolvendo profissionais de saúde de diversas áreas do conhecimento, tal como farmacêuticos (Faria et al., 2018).

Atualmente, a atuação do farmacêutico em oncologia é uma realidade presente em praticamente todos os serviços de quimioterapia pelo Brasil. Suas atribuições excedem a simples dispensação da prescrição médica, ou ainda a manipulação propriamente dita, uma vez que, sua atuação é importante em várias etapas da terapia antineoplásica, inclusive sua participação em diversas comissões (Farmácia e Terapêutica, Infecção Hospitalar, Biossegurança). Constituídas por equipe multiprofissional, que se reúne para tomar decisões, com objetivo de garantir assistência integral ao paciente oncológico (Do Amaral, 2018).

\section{Metodologia}

O presente estudo consiste em uma revisão integrativa de literatura (RIL), por ser muito utilizado na análise de conceitos, revisão de teorias ou evidências síntese do conhecimento sobre determinado tema, permitindo identificar lacunas que precisam ser preenchidas com a realização de novos estudos (Aguiar et al., 2020). Esta RIL discorre sobre as interações medicamentosas em pacientes com câncer de mama.

Para realizar essa revisão integrativa, considerou-se os estudos de Yamagata et al. (2018) e Batista et al. (2021), sendo percorridas as seguintes etapas: definição do tema, elaboração da pergunta norteadora, estabelecimento de critérios de busca ou amostragem na literatura, definição das informações extraídas dos artigos (coleta de dados), análise crítica dos artigos incluídos, discussão e apresentação dos resultados.

Para nortear a revisão integrativa, elaborou-se a seguinte questão: É frequente a ocorrência de interações medicamentosas em pacientes com câncer de mama? A pesquisa foi realizada nos meses de agosto e setembro de 2021 nas bases de dados descritas abaixo, utilizando os Descritores de Ciências da Saúde (DeCS). Os descritores selecionados foram "Interações de Medicamentos", "câncer de mama" e "Brasil". A busca por artigos originais considerou os conectores boleanos: "drug interactions and breast cancer and brazil".

As pesquisas foram realizadas utilizando as bases de dados: PubMed, Scientific Eletronic Library (Scielo) e Biblioteca Virtual em Saúde (BVS). Utilizou-se como critérios de inclusão artigos com resumo e texto completos disponíveis eletronicamente, escritos em português ou inglês, realizados no Brasil, não havendo restrições quanto ao ano de publicação dos trabalhos, em periódicos científicos e que estivessem adequados ao tema proposto para o trabalho. As estratégias de busca foram executadas distintamente devido às particularidades de cada base de dados. A pesquisa não se limitou apenas a artigos, podendo ser inseridos documentos acadêmicos, caso estivessem disponíveis nas bases de dados consultadas. Não foi realizada busca em literatura cinzenta.

Foram excluídos do estudo, artigos que não abordaram, em conjunto, os temas: interação medicamentosa em pacientes com câncer de mama, artigos que abordaram esses aspectos, porém, em outras doenças, que não o câncer; artigos duplicados nas bases de dados, artigos que não estavam disponíveis na íntegra, com acesso restrito a pagamento.

O processo de seleção de trabalhos para o presente artigo foi realizado por meio de revisão por pares, de forma independente. As divergências foram discutidas entre os dois autores até se obter um consenso dos artigos que, de fato, seriam selecionados e analisados. 
Dos trabalhos incluídos nesta revisão integrativa, foram extraídas as seguintes informações: autoria, ano de publicação, idioma da publicação, tipo de estudo, tempo de pesquisa (duração da coleta dos dados), amostra (número de pacientes/interações, artigos ou de prescrições), faixa etária, abordagem das interações (IM) presentes, fonte de informação utilizada para descrevê-las, se o estudo foi geral ou específico para as IM, fonte para coleta de dados e localidade da execução do trabalho.

Por tratar-se de um estudo de revisão da literatura este trabalho não foi submetido a um Comitê de Ética em Pesquisa (CEP).

\section{Resultados e Discussão}

Na busca foram encontrados 28 artigos, após as diferentes etapas, foram selecionados para esta revisão 9 trabalhos, conforme fluxograma apresentado na Figura 1. Tendo em vista os trabalhos selecionados, observou-se que de acordo com a revisão integrativa, 4 dos artigos foram publicados em inglês e 5 em português. O quadro 1 sumariza os dados relativos aos artigos incluídos nesta revisão.

Figura 1: Fluxograma da busca de artigos nas bases de dados.

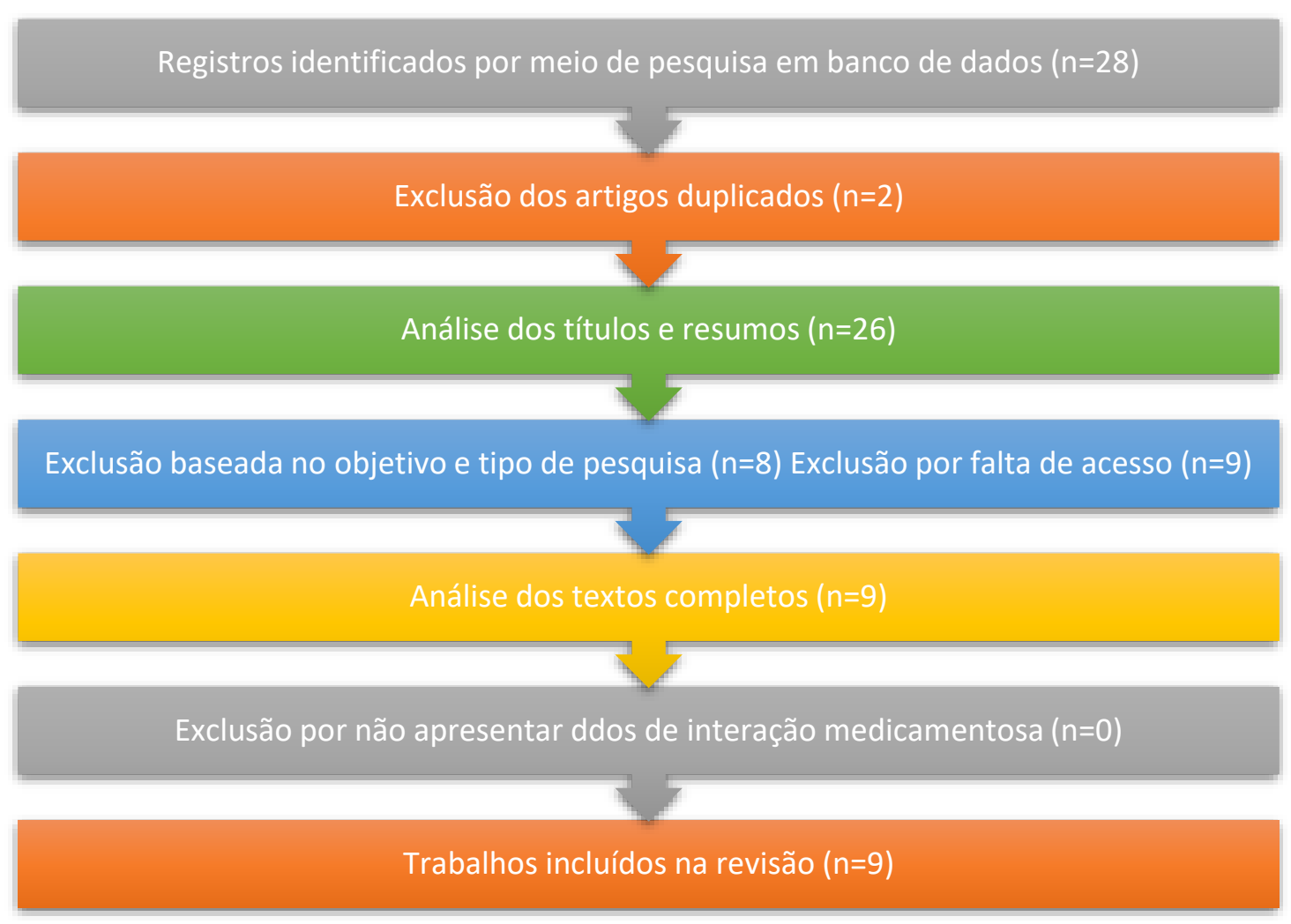

Fonte: Autores (2021). 
Research, Society and Development, v. 10, n. 16, e39101621243, 2021

(CC BY 4.0) | ISSN 2525-3409 | DOI: http://dx.doi.org/10.33448/rsd-v10i16.21243

\begin{tabular}{|c|c|c|c|c|c|c|}
\hline \multicolumn{7}{|c|}{ Quadro 1: Resumo dos artigos levantados nas bases de Dados. } \\
\hline Autores & Título & $\begin{array}{l}\text { Local de } \\
\text { busca }\end{array}$ & $\begin{array}{l}\text { Tipo de } \\
\text { pesquisa }\end{array}$ & Descritores & $\begin{array}{l}\text { Ano de } \\
\text { publicação }\end{array}$ & Conclusões/Recomendações \\
\hline 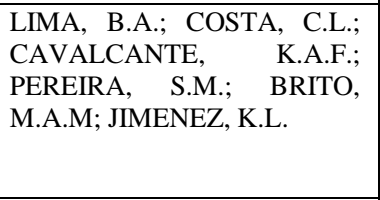 & $\begin{array}{lccr}\text { Desenvolvimento } & \text { de } & \text { protocolo } & \text { de } \\
\text { acompanhamento } & \text { farmacoterapêutico } & \text { a } \\
\text { pacientes em tratamento de câncer de mama } & \end{array}$ & Pubmed & $\begin{array}{l}\text { Revisão } \\
\text { bibliográfica }\end{array}$ & $\begin{array}{l}\text { "Interações de } \\
\text { Medicamentos", "câncer } \\
\text { de mama" e "Brasil" }\end{array}$ & 2021 & $\begin{array}{l}\text { O estudo conclui que seguimento } \\
\text { farmacoterapêutico visa alcançar resultados que } \\
\text { melhorem a qualidade de vida dos pacientes. } \\
\text { Detectar e resolver os problemas que apareçam no } \\
\text { tratamento oncológico, de forma sistematizada e } \\
\text { documentada é de suma importância. }\end{array}$ \\
\hline $\begin{array}{lr}\text { MORIEL, P.; } & \text { SIQUEIRA, } \\
\text { J.A.; CARNEVALE, } & \text { R.C.; } \\
\text { COSTA, C.G.R.; DA CRUZ, } \\
\text { A.A.; DA SILVA, N.M.O; } \\
\text { BERNARDES, } & \text { A.C.; } \\
\text { CARVALHO, } & \text { R.P; } \\
\text { MAZZOLA, P.G. } & \\
\end{array}$ & $\begin{array}{l}\text { Drug interactions in female oncologic } \\
\text { inpatients: differences among databases }\end{array}$ & BVS & $\begin{array}{l}\text { Descritivo } \\
\text { crossover }\end{array}$ & $\begin{array}{l}\text { "Interações de } \\
\text { Medicamentos", "câncer } \\
\text { de mama" e "Brasil" }\end{array}$ & 2013 & $\begin{array}{l}\text { Considera-se necessária uma padronização nas } \\
\text { ferramentas de análise de interações, além da análise } \\
\text { criteriosa das prescrições por um farmacêutico junto } \\
\text { à equipe multidisciplinar, a fim de promover práticas } \\
\text { seguras no uso dos medicamentos, reduzindo, desta } \\
\text { forma, possíveis complicações causadas por } \\
\text { interações medicamentosas. }\end{array}$ \\
\hline $\begin{array}{lr}\text { CUPERTINO, } & \text { A.; } \\
\text { MARCONDES, } & \text { M.A.; } \\
\text { GATTI, R.M. } & \end{array}$ & $\begin{array}{l}\text { Estudo retrospectivo das reações adversas e } \\
\text { interações medicamentosas na quimioterapia } \\
\text { no tratamento do câncer de mama: relato de } \\
\text { caso }\end{array}$ & Pubmed & Relato de caso & $\begin{array}{l}\text { "Interações de } \\
\text { Medicamentos", "câncer } \\
\text { de mama" e "Brasil" }\end{array}$ & 2008 & $\begin{array}{l}\text { No caso clínico acompanhado, verificaram-se as } \\
\text { reações adversas causadas pelos medicamentos, e } \\
\text { que a associação de fluorouracil e ciclofosfamida } \\
\text { pode estar associada ao aumento da toxicidade } \\
\text { hematológica, além disso, recomendam que o } \\
\text { farmacêutico seja incorporado na equipe que } \\
\text { acompanha o usuário. }\end{array}$ \\
\hline $\begin{array}{lll}\text { FARIA, C.O.; } & \text { REIS, } & \text { C.M.; } \\
\text { SANTOS, } \quad \text { A.G.; } & \text { REIS, } \\
\text { A.M.M. } & & \end{array}$ & $\begin{array}{l}\text { Interações Medicamentosas na Farmacoterapia } \\
\text { de Idosos com Câncer atendidos em um } \\
\text { Ambulatório de Onco-Hematologia }\end{array}$ & Pubmed & Transversal & $\begin{array}{l}\text { "Interações de } \\
\text { Medicamentos", "câncer } \\
\text { de mama" e "Brasil" }\end{array}$ & 2018 & $\begin{array}{l}\text { No presente estudo, a frequência de interações } \\
\text { fármaco--fármaco foi elevada, acometendo } \\
\text { aproximadamente dois terços dos idosos. Observou- } \\
\text { se ampla variabilidade na frequência de interações } \\
\text { com resultados entre } 16 \% \text { e } 75 \% \text {. Destaca-se a } \\
\text { prevalência em torno de } 50 \% \text { de interações } \\
\text { classificadas como graves. }\end{array}$ \\
\hline GARCIA, H.O & $\begin{array}{l}\text { Efeito da interação medicamentosa entre } \\
\text { tamoxifeno e antidepressivos inibidores da } \\
\text { cyp2d6 na morbimortalidade de mulheres com } \\
\text { câncer de mama: uma revisão sistemática. }\end{array}$ & BVS & $\begin{array}{l}\text { Revisão } \\
\text { sistemática }\end{array}$ & $\begin{array}{l}\text { "Interações de } \\
\text { Medicamentos", "câncer } \\
\text { de mama" e "Brasil" }\end{array}$ & 2021 & $\begin{array}{l}\text { O risco de recorrência e de morte em pacientes com } \\
\text { câncer de mama tratadas com tamoxifeno não parece } \\
\text { ser associado com o uso de antidepressivos } \\
\text { inibidores da CYP2D6 até o momento. }\end{array}$ \\
\hline $\begin{array}{l}\text { DO AMARAL, } \text { P. } \\
\text { MENDONÇA, S.; } \\
\text { OLIVEIRA, D.R. ; PELOSO, } \\
\text { L.J. ; PEDROSO, R. S.; } \\
\text { RIBEIRO, M. A. }\end{array}$ & $\begin{array}{l}\text { Impact of a medication therapy management } \\
\text { service offered to patients in treatment of } \\
\text { breast câncer. }\end{array}$ & Pubmed & $\begin{array}{l}\text { Observacional, } \\
\text { exploratório, } \\
\text { descritivo e } \\
\text { retrospectivo }\end{array}$ & $\begin{array}{l}\text { "Interações } \quad \text { de } \\
\text { Medicamentos", "câncer } \\
\text { de mama" e "Brasil" }\end{array}$ & 2018 & $\begin{array}{l}\text { O estudo enfatiza o impacto positivo da adoção do } \\
\text { serviço de acompanhamento farmacoterapeutico, } \\
\text { fato que leva a uma resolução de um grande número } \\
\text { de problemas relacionados a medicamentos. Sugere } \\
\text { que haja uma prática multidisciplinar e } \\
\text { interdisciplinar do cuidado. }\end{array}$ \\
\hline $\begin{array}{lr}\text { ANTUNES, } & \text { M.V.;TIMM, } \\
\text { T.A.F.; DE } & \text { OLIVEIRA, V.; } \\
\text { STAUDT, } & \text { D.E.; } \\
\text { RAYMUNDO, S. ; } & \text { Gustavo } \\
\text { GÖSSLING, } & \text { G.; } \\
\text { BIAZÚS,J.V.; } & \\
\end{array}$ & $\begin{array}{l}\text { Influence of CYP2D6 and CYP3A4 } \\
\text { Phenotypes, Drug Interactions, and Vitamin D } \\
\text { Status on Tamoxifen Biotransformation. }\end{array}$ & Pubmed & Analítico & $\begin{array}{l}\text { "Interações de } \\
\text { Medicamentos", "câncer } \\
\text { de mama" e "Brasil" }\end{array}$ & 2015 & $\begin{array}{l}\text { A vitamina } \mathrm{D} \text { não foi associada a atividade } \\
\text { metabólica do CYP3A4. }\end{array}$ \\
\hline
\end{tabular}


Research, Society and Development, v. 10, n. 16, e39101621243, 2021

(CC BY 4.0) | ISSN 2525-3409 | DOI: http://dx.doi.org/10.33448/rsd-v10i16.21243

\begin{tabular}{|c|c|c|c|c|c|}
\hline $\begin{array}{l}\text { CAVALHEIRO, J.A.; ROSA, } \\
\text { D.D.; WALLEMACQ,P.; } \\
\text { HAUFROID, V.; LINDEN, R.; } \\
\text { SCHWARTSMANN,G. }\end{array}$ & & & & & \\
\hline $\begin{array}{lrr}\text { LOBO, } & \text { R.E.D.; } & \text { BAHIA, } \\
\text { B.P.G; } & \text { SILVA, } & \text { G.E.A.; } \\
\text { CRUZ, L.N.; SARGES, E.S.; } \\
\text { LO } & \text { PRETE, } & \text { A.C.; } \\
\text { CARNEIRO, T.X.; RIBEIRO, } \\
\text { C.H.M.A }\end{array}$ & $\begin{array}{l}\text { Interação medicamentosa em pacientes com } \\
\text { câncer: revisão integrativa da literatura. }\end{array}$ & $\begin{array}{l}\text { Revisão } \\
\text { integrativa }\end{array}$ & $\begin{array}{l}\text { "Interações de } \\
\text { Medicamentos", "câncer } \\
\text { de mama" e "Brasil" }\end{array}$ & 2021 & $\begin{array}{l}\text { Esta revisão demonstrou uma alta prevalência de } \\
\text { interações medicamentosas devido à complexidade } \\
\text { da farmacoterapia em pacientes adultos tratados com } \\
\text { quimioterápicos. Esses dados sugerem que é } \\
\text { essencial que se adote métodos para minimização de } \\
\text { possíveis potenciais interações medicamentosas. O } \\
\text { conhecimento das interações mais comuns na prática } \\
\text { clínica é uma ferramenta essencial para criação de } \\
\text { estratégias de prevenção. }\end{array}$ \\
\hline $\begin{array}{l}\text { FERRACINI, } r \text { A.C.; } \\
\text { RODRIGUES, A.T.; DE } \\
\text { BARROS, A.A.; DERCHAIN, } \\
\text { S.F.; MAZZOLA, P.G. }\end{array}$ & $\begin{array}{l}\text { Prescribing errors intercepted by pharmacist } \\
\text { intervention in care of patients hospitalised } \\
\text { with breast and gynaecological cancer at a } \\
\text { Brazilian teaching hospital }\end{array}$ & $\begin{array}{l}\text { Transversal } \\
\text { prospectivo }\end{array}$ & $\begin{array}{l}\text { "Interações de } \\
\text { Medicamentos", "câncer } \\
\text { de mama" e "Brasill" }\end{array}$ & 2017 & $\begin{array}{l}\text { Conclui que o principal erro de prescrição é a } \\
\text { interação medicamentosa com medicamentos para o } \\
\text { trato gastrointestinal, metabolismo e anti-infeccioso } \\
\text { de uso sistêmico. Relatam que as intervenções } \\
\text { farmacêuticas realizadas tiveram impacto } \\
\text { significativo para evitar os erros de prescrição. }\end{array}$ \\
\hline
\end{tabular}

Fonte: Autores (2021). 
Na maioria dos artigos analisados, a interação medicamentosa em pacientes com câncer, apresenta-se como principal problema relacionado a medicamentos. Em todos conseguimos perceber a importância de se monitorar as prescrições e trabalhar em conjunto com a equipe multiprofissional. Portanto, os estudos reafirmam que as interações medicamentosas variam muito de paciente para paciente, tal como a gravidade das consequências de interações. Essa variação depende principalmente de fatores como idade, constituição genética, estado patológico, tempo do tratamento e alimentação. Portanto, cabe avaliar o risco e o benefício em cada caso estudado.

Seguindo esse raciocínio, Lima et al. (2021), em sua revisão bibliográfica, observou que pacientes com câncer de mama em tratamento com antineoplásicos tem uma grande possibilidade de ter complicações no tratamento, pois os problemas relacionados a medicamentos mais identificados foram erros de medicação, eventos adversos e interação medicamentosa, podendo comprometer a adesão ao tratamento, corroborando com Lobo et al. (2021), que também demonstrou uma alta prevalência de interações medicamentosa devido à complexidade da farmacoterapia.

Além disso, os estudos de Lima et al. (2021) e Dominguez-Alonso et al. (2021), corroboram com os estudos de Amaral et al. (2018) onde estes detectaram cerca de 69,7\% de interações medicamentosas e alimentares. A maioria destes PRM foi resolvida entre farmacêutico e paciente $(61,86 \%)$, sendo necessário em alguns casos, o encaminhamento das pacientes para outros profissionais. Alinhado a estes dois estudos, Silva et al. (2020) sugere a participação do profissional farmacêutico, sendo proposto por Lima et al. (2021) um plano de cuidado farmacêutico, pois isso garante o uso racional e seguro de medicamentos antineoplásicos, sendo essencial para detectar as interações medicamentosas, melhorando a conciliação terapêutica.

Outro ponto a considerar dentro da visão do cuidado e rastreamento de IM, é o mostrado por Lobo et al. (2021) e Amaral et al. (2018), os quais sugerem a criação de serviço de monitoramento da terapia medicamentosa em pacientes com câncer de mama, havendo o incentivo ao cuidado multiprofissional e interdisciplinar. Amaral et al. (2018) e Irarrázaval e Gaete (2016) relatam que a principal causa de PRM em relação a eficácia foi a dosagem muito baixa, fato que resulta de interações medicamentosas e alimentares, e cita como exemplo a interação entre fluoxetina e tamoxifeno.

Outros exemplos de interação também foram abordados: o estudo de Moriel et al. (2013) objetivou quantificar as interações medicamentosas em prescrições de mulheres submetidas à terapia de suporte no setor de oncologia de um hospital universitário brasileiro especializado na saúde da mulher e comparar as informações fornecidas por diferentes bases de dados em relação a estas interações medicamentosas. Analisando este estudo, verificou-se um total de 152 interações medicamentosas, sendo as principais: haloperido/amitriptilina (32,4\%), codeína/diazepam $(11,8 \%)$ em uma enfermaria e captopril/cloreto de potássio $(33,3 \%)$ e diazepam/morfina (25\%) em outra enfermaria. Além disso, outras interações como entre captopril/ringer lactato e cloreto de potássio/escopolamina também requerem monitoramento. Rodrigues et al. (2020) comunga com a conclusão deste estudo no que se refere a discrepância entre os instrumentos de avaliação das interações, sugerindo uma padronização e a análise criteriosa das prescrições por um farmacêutico junto à equipe multidisciplinar.

Não somente o artigo citado acima relata a importância de se estudar as IM, o estudo de Cupertino et al. (2008) é um relato de caso que aborda o tratamento quimioterápico feito em uma mulher de 47 anos de idade, com diagnóstico de câncer invasivo lobular da mama direita. A qual fez uso do sistema quimioterápico 5-fluoracil, ciclofosfamida e epirrubicina, durante o período de ag0osto a dezembro de 2006. Com o objetivo de verificar as reações adversas e as possíveis interações medicamentosas, foram analisadas as bulas de cada medicamento, comparando-as com as reações adversas que a paciente apresentou e as interações indicadas por cada um dos medicamentos utilizados. No caso clínico acompanhado, verificaram-se as reações adversas causadas pelos medicamentos, podendo ter sido intensificada devido à associação de epirrubicina com fluorouracil e ciclofosfamida, causando um aumento da mielossupressão, caracterizado por diminuição dos níveis de leucócitos 
e linfócitos da paciente. A associação de fluorouracil e ciclofosfamida pode estar relacionada ao aumento da toxicidade hematológica apresentada por ela.

Fluorouracil também é citado no estudo de Farias et al. (2018) que analisaram a frequência das IM e fizeram análise dos fatores associados na farmacoterapia. Noventa e sete (60,6\%) idosos apresentaram pelo menos uma interação medicamentosa e $42(26,3 \%)$ interação envolvendo um medicamento antineoplásico. Ciclofosfamida e fluouracila foram os mais envolvidos em interações. O mecanismo farmacodinâmico foi responsável por 52,5\% das interações. Identificou-se que $51 \%$ das interações foram classificadas como graves. O estudo concluiu que a frequência de interações medicamentosas potenciais em idosos com câncer em terapia antineoplásica parenteral foi elevada, corroborando com Amaral et al. (2018).

Percebeu-se que, tanto em Amaral et al. (2018) quanto em Garcia (2021), o fármaco tamoxifeno ganha destaque. Este último autor, em uma revisão sistemática, investigou a interação medicamentosa entre Tamoxifeno e antidepressivos inibidores da CYP2D6. Dos nove estudos incluídos no trabalho, um identifica um risco aumentado na mortalidade e outro na recorrência de pacientes usuárias de paroxetina e tamoxifeno. Não foram encontrados riscos aumentados para outros antidepressivos, independentemente da força de inibição. Outros autores que seguiram essa mesma linha são:Antunes et al. (2015) analisaram a interação medicamentosa e exposição à vitamina D no metabolismo do tamoxifeno através de amostras de sangue de pacientes oncológicas, concluíram que cerca de 20\% destas tiveram redução da atividade da enzima CYP2D6 e 7\% do metabolismo da CYP3A4 também reduzido.

Ferracini et al. (2017), através de análise de prescrições, registraram os erros presentes nelas e categorizaram as mesmas. As IM foram categorizadas como inseguras, logo as intervenções farmacêuticas realizadas foram voltadas mais para esse problema. O presente estudo confirma que os erros nas prescrições para pacientes hospitalizados com câncer de mama e ginecológico foram principalmente associados a IM. O estudo também registrou as principais classes de medicamentos envolvidas nos erros de prescrição: os medicamentos para o trato alimentar e metabolismo, os anti-infecciosos de uso sistêmico e os medicamentos para o sangue e órgãos hematopoiéticos.

Considerando os erros de prescrição e a importância da minimização destes, Lima et al. (2021), Moriel et al. (2013) e Ferracini et al. (2017), destacam que o profissional farmacêutico quando inserido na equipe multidisciplinar, desempenha um papel importante no cuidado a pacientes em tratamento de câncer de mama, pois este pode identificar potenciais barreiras ao uso apropriado de medicamentos, assim como também pode afetar significativamente os eventos adversos a estes medicamentos, através de sugestão de redução de dose, educação a paciente quanto à quimioterapia oral e participação na diminuição das reações adversas. Através destes estudos, foi possível concluir que os farmacêuticos contribuem para reduzir eventos adversos como náuses e mal-estar, contribuindo, assim, para melhoria da qualidade de vida das pacientes.

\section{Conclusão}

Com base no levantamento de dados, observou-se que a intervenção farmacêutica aumentou a identificação e resolução de PRM e de erros na utilização de medicamentos, tal como diminuiu a possibilidade de IM em mulheres com câncer de mama.

Estabelecer uma rotina para identificação de potenciais interações medicamentosas, de forma integrada entre a farmácia e as equipes de enfermagem e de prescritores, pode favorecer a prevenção de IM potenciais, nos vários níveis de gravidade, já que mesmo pequenas alterações na farmacocinética ou farmacodinâmica de um agente quimioterápico podem trazer consequências significativas na sua eficácia ou toxicidade e, assim, interferir no sucesso do tratamento. Em geral, dificilmente o médico conhece todas as possíveis interações medicamentosas existentes e as suas consequências clínicas. A presença do farmacêutico pode estabelecer o controle regular deste tipo de interações. 
Nesse contexto, o papel do farmacêutico é fundamental para uma melhora na qualidade de vida desses pacientes, pois ele pode esclarecer dúvidas quanto aos medicamentos, quanto a sua doença, pode viabilizar meios para a adesão ao tratamento medicamentoso, orientar quanto à necessidade, os riscos e os benefícios da medicação e promover o uso racional desses medicamentos. Trabalhos futuros de acompanhamento farmacoterapêutivo são importantes para um melhor desfecho da qualidade de vida de todos os pacientes.

\section{Referências}

Amaral, P. A., Mendonça, S. M., Oliveira, D. R., Peloso, L.J., Pedroso, R. S., \& Ribeiro, M. A. (2018). Impact of a medication therapy management service offered to patients in treatment of breast câncer. Brazilian Journal of Pharmaceutical Science: 1-12. http://dx.doi.org/10.1590/s2175-97902018000200221.

Aguiar, R. B., Leal, M. C.C., Marques, A. P. de O., Torres, K. M. S., \& Tavares, M. T. D. B. (2020) Idosos vivendo com HIV - comportamento e conhecimento sobre sexualidade: revisão integrativa. Ciência \& Saúde Coletiva: 575-584. https://doi.org/10.1590/1413-81232020252.12052018.

Antunes, M.V., Timm, T. A. F., De Oliveira, V., Staudt, D. E., Raymundo, S., Gössling, G., Biazús, J.V., Cavalheiro, J. A., Rosa, D. D., Wallemacq, P., Haufroid, V., Linden, R., \& Schwartsmann, G. (2015). Influence of CYP2D6 and CYP3A4 Phenotypes, Drug Interactions, and Vitamin D Status on Tamoxifen Biotransformation. Ther Drug Monit:733-744. https://pubmed.ncbi.nlm.nih.gov/25853922/.

Batista, A.V.A., Dos Santos, V. R. C., \& Carneiro, I. C. R. S. (2021). Cuidado farmacêutico em oncologia: Revisão integrativa da literatura. Research, Society and Development:1-14. https://rsdjournal.org/index.php/rsd/article/download/14987/13497/195839.

Cupertino, A., Marcondes, M. A., \& Gatti, R. M. (2008). Estudo retrospectivo das reações adversas e interações medicamentosas na quimioterapia no tratamento do câncer de mama: relato de caso. Revista Brasileira de Ciências da Saúde: 26-36. https://seer.uscs.edu.br/index.php/revista_ciencias_saude/article/view/356.

Domínguez-Alonso, J.A., Conde-Estévez, D., Bosch, D. Pi-Figueras, M., \& Tuquets, I. (2021). Breast cancer, placing drug interactions in the spotlight: is polypharmacy the cause of everything? Clinical and Translational Oncology: 23, 65-73 (2021). Disponível em: https://doi.org/10.1007/s12094-020-02386-8.

Faria, C. O., Reis, C. M., Santos, A. G., \& Reis, A. M. M. (2018). Interações Medicamentosas na Farmacoterapia de Idosos com Câncer atendidos em um Ambulatório de Onco-Hematologia. Revista Brasileira de Cancerologia: 61-68. https://rbc.inca.gov.br/revista/index.php/revista/article/view/119.

Ferracini, A. C., Rodrigues, A. T., De Barros, A. A., Derchain, S. F., \& Mazzola, P. G. (2017). Prescribing errors intercepted by pharmacist intervention in care of patients hospitalised with breast and gynaecological cancer at a Brazilian teaching hospital. European Journal Cancer Care:1-7. https://pubmed.ncbi.nlm.nih.gov/28925569/.

Garcia, H. O. Efeito da interação medicamentosa entre tamoxifeno e antidepressivos inibidores da cyp2d6 na morbimortalidade de mulheres com câncer de mama: uma revisão sistemática. Porto Alegre. 2021. Trabalho de Conclusão de Curso (Faculdade de Farmácia), Universidade Federal do Rio Grande do Sul. Porto Alegre, 2021. http://hdl.handle.net/10183/225671.

Instituto Nacional do Câncer (INCA/MS). Tipos de câncer: Câncer de mama. 2020. Disponível em: https://www.inca.gov.br/estimativa/sintese-deresultados-ecomentários.

Irarrázaval, M. E., \& Gaete, L. (2016). Elección del mejor antidepresivo en pacientes con cáncer de mama en tratamiento con tamoxifeno: revisión de la evidencia básica y clínica. Revista Médica de Chile:1326- 1335. https://www.scielo.cl/scielo.php?script=sci_arttext\&pid=S0034$98872016001000013 \& \operatorname{lng}=$ en $\&$ nrm=iso\&tlng=en.

Lima, B. A., Costa, C. L., Cavalcante, K. A. F., Pereira, S. M., Brito, M. A. M., \& Jimenez, K. L. (2021). Desenvolvimento de protocolo de acompanhamento farmacoterapêutico a pacientes em tratamento de câncer de mama. Brazilian Journal of Health Review: 11321-11340. https://www.brazilianjournals.com/index.php/BJHR/article/view/30258.

Lobo, R. E. D., Bahia, B. P. G., Silva, G. E. A., Cruz, L. N., Sarges, E. S., Lo Prete, A. C., Carneiro, T. X., \& Ribeiro, C. H. M. A. (2021) Interação medicamentosa em pacientes com câncer: revisão integrativa da literatura. Brazilian Journal of Development: $32289-32303$. https://www.brazilianjournals.com/index.php/BRJD/article/view/27304.

Machado, M. X., Soares, D. A., \& Oliveira, S. B. (2017). Significados do câncer de mama para mulheres no contexto do tratamento quimioterápico. Physis Revista de Saúde Coletiva: 433-451. https://doi.org/10.1590/S0103-73312017000300004.

Moriel, P., Siqueira, J. A., Carnevale, R. C., Costa, C. G. R., Da Cruz, A. A., Da Silva, N. M. O., Bernardes, A. C., Carvalho, R. P., \& Mazzola, P. G. (2013). Drug interactions in female oncologic inpatients: differences among databases. Revista de Ciências Farmacêuticas Básica e Aplicada: 333-337. https://rcfba.fcfar.unesp.br/index.php/ojs/article/view/193

Quemel, G. K. C., Da Silva, E. P., Conceição, W. R., Gomes, M. F., Rivera, J. G. B., \& Quemel, G. K, C. (2018). Revisão integrativa da literatura sobre o aumento no consumo de psicotrópicos em transtornos mentais como a depressão. Brazilian Applied Science Review:1384-1403. https://www.brazilianjournals.com/index.php/BASR/article/view/30182

Rodrigues, H. S., Souza, R. P., Sousa, R. W. R. de, Pereira, L. C. A., Branco, R. R. de O. C., Silveira, P. H. F. P., \& Britto, M. H. R. M. (2020). Drug interactions profile of Oral Antineoplastic Agents (OAAs) dispensed for oncologic patients. Research, Society and Development, 9(8), e145985369. https://doi.org/10.33448/rsd-v9i8.5369 
Research, Society and Development, v. 10, n. 16, e39101621243, 2021

(CC BY 4.0) | ISSN 2525-3409 | DOI: http://dx.doi.org/10.33448/rsd-v10i16.21243

Silva, G. G. S., Silva, J. A., Souza, E. B., Gomes, S. A. C., Santana, N. B. L., Galindo, J. A., Tenório, E. M. A., Melo, R. K. S., Souza, A. P., \& Barros, Danilo, P. O. (2020). Importância do farmacêutico clínico na diminuição das interações medicamentosas ao paciente oncológico na unidade de terapia intensiva. Brazilian Journal of health Review: 3 (5) 15542-15556.

Topaloğlu, U. S., \& Özaslan, E. (2020). Comorbidity and polypharmacy in patients with breast cancer. Breast Cancer: 27, 477-482. https://doi.org/10.1007/s12282-019-01040-8

Yamagata, A. T., Coelho, R. M. P., Júnior, B., Galato, D., Meiners, M. M. M. A., \& Da Silva, E. V. (2018). Perfil dos estudos de interações medicamentosas potenciais em hospitais brasileiros: revisão integrativa. Revista Brasileira de Farmácia Hospitalar e Serviços de Saúde: 1-9. https://www.rbfhss.org.br/sbrafh/article/download/386/375 